
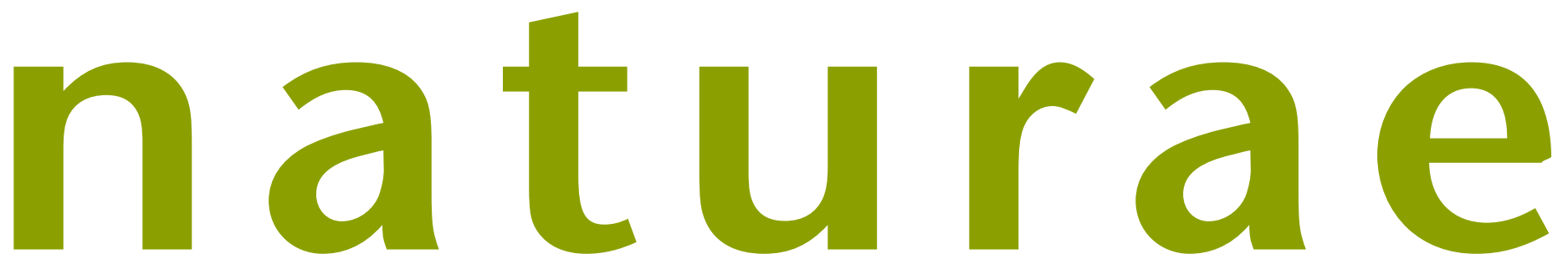

$2021 \cdot 7$

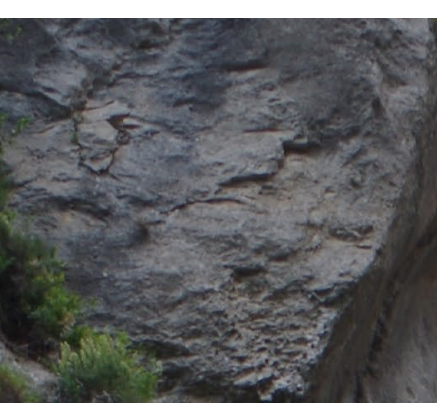

8. P. Des méthodes pour évaluer -1 l'état de conservation des habitats humides
sur Kensemble du territoire métropolitain -1 l'état de conservation des habitats humides
sur Kensemble du territoire métropolitain
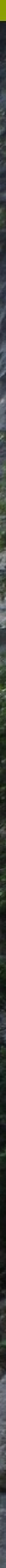
Directeur de LA Publication / Publication diRECTOR: Bruno David,

Président du Muséum national d'Histoire naturelle

RÉDACTEUR EN CHEF / EDITOR-IN-CHIEF: Jean-Philippe Siblet

ASSISTANTE DE RÉDACTION / ASSISTANT EDITOR: Sarah Figuet (naturae@mnhn.fr)

Mise EN PAGE / PAGE LAYOUT: Sarah Figuet

COMITÉ SCIENTIFIQUE / SCIENTIFIC BOARD:

Luc Abbadie (UPMC, Paris)

Luc Barbier (Parc naturel régional des caps et marais d'Opale, Colembert)

Aurélien Besnard (CEFE, Montpellier)

Vincent Boullet (Expert indépendant flore/végétation, Frugières-le-Pin)

Hervé Brustel (École d'ingénieurs de Purpan, Toulouse)

Patrick De Wever (MNHN, Paris)

Thierry Dutoit (UMR CNRS IMBE, Avignon)

Éric Feunteun (MNHN, Dinard)

Romain Garrouste (MNHN, Paris)

Grégoire Gautier (DRAAF Occitanie, Toulouse)

Olivier Gilg (Réserves naturelles de France, Dijon)

Frédéric Gosselin (Irstea, Nogent-sur-Vernisson)

Patrick Haffner (UMS PatriNat, Paris)

Frédéric Hendoux (MNHN, Paris)

Xavier Houard (OPIE, Guyancourt)

Isabelle Le Viol (MNHN, Concarneau)

Francis Meunier (Conservatoire d'espaces naturels - Hauts-de-France, Amiens)

Serge Muller (MNHN, Paris)

Francis Olivereau (DREAL Centre, Orléans)

Laurent Poncet (UMS PatriNat, Paris)

Nicolas Poulet (OFB, Vincennes)

Jean-Philippe Siblet (UMS PatriNat, Paris)

Laurent Tillon (ONF, Paris)

Julien Touroult (UMS PatriNat, Paris)

COUVERTURE / COVER:

Cascade de tuf et végétation associée dans les Alpes-Maritimes, habitat humide d'intérêt communautaire (UE 7220*). Crédit photo: M. Mistarz.

Naturae est une revue en flux continu publiée par les Publications scientifiques du Muséum, Paris

Naturae is a fast track journal published by the Museum Science Press, Paris

Les Publications scientifiques du Muséum publient aussi / The Museum Science Press also publish:

Adansonia, Zoosystema, Anthropozoologica, European Journal of Taxonomy, Geodiversitas, Cryptogamie sous-sections Algologie, Bryologie, Mycologie, Comptes Rendus Palevol.

Diffusion - Publications scientifiques Muséum national d'Histoire naturelle

CP $41-57$ rue Cuvier F-75231 Paris cedex 05 (France)

Tél.: 33 (0)140794805 / Fax: 33 (0)1 40793840

diff.pub@mnhn.fr / http://sciencepress.mnhn.fr

(C) Publications scientifiques du Muséum national d'Histoire naturelle, Paris, 2021

ISSN (électronique / electronic): 1638-9387 


\title{
Des méthodes pour évaluer l'état de conservation des habitats humides sur l'ensemble du territoire métropolitain
}

\author{
Margaux MISTARZ \\ UMS PatriNat, \\ Office français de la biodiversité, CNRS, Muséum national d'Histoire naturelle, \\ 4 avenue du petit château, F-91800 Brunoy (France) \\ margaux.mistarz@mnhn.fr
}

Soumis le 29 septembre 2020 | Accepté le 19 novembre 2020 | Publié le 17 mars 2021

MOTS CLÉS

Directive Habitats-

Faune-Flore,

Natura 2000

de l'environnement,

gestion.

Mistarz M. 2021. - Des méthodes pour évaluer l'état de conservation des habitats humides sur l'ensemble du territoire métropolitain. Naturae 2021 (7): 79-90. https://doi.org/10.5852/naturae2021a7

\section{RÉSUMÉ}

Un des objectifs de la directive Habitats-Faune-Flore est le maintien, ou la restauration, dans un état de conservation (EC) favorable des habitats et des espèces d'intérêt communautaire (HIC) listés dans ses annexes. Afin de répondre à cet objectif, le ministère en charge de l'Écologie a confié au Muséum national d'Histoire naturelle la mise en place de méthodes d'évaluation de l'EC des HIC à l'échelle des sites Natura 2000. Ces méthodes doivent être simples, reproductibles et standardisées sur l'ensemble du territoire métropolitain. Les travaux sont engagés depuis 2008 et ont vu paraître plusieurs guides méthodologiques traitant des habitats forestiers, dunaires, agropastoraux, marins et lagunaires. Pour les habitats humides, un premier travail a porté sur les eaux courantes en 2013. Se sont succédés depuis plusieurs travaux, notamment sur les eaux dormantes, les habitats tourbeux ou encore les landes humides atlantiques. Cet article vise à présenter le principe méthodologique de l'évaluation de l'EC à l'échelle des sites, ainsi que le processus d'élaboration des grilles d'évaluation. Il vise également à faire le bilan de l'ensemble des méthodes disponibles à ce jour pour évaluer l'EC des HIC humides. À l'heure actuelle, 98 \% des HIC humides recensés sur le territoire métropolitain sont couverts par une méthode d'évaluation ou ont fait l'objet d'une première approche méthodologique.

\begin{abstract}
Methods to assess the conservation status of wet habitats throughout mainland France.

The Habitats Directive is the first normative document that has ever defined the notion of "conservation status". The objective is to maintain or restore the habitats of Community Interest in a favourable conservation status. These types of habitats are listed in the Directive (Annex I). Thus, a network of protected areas called "Natura 2000" has been established. Special Areas of Conservation (SACs, Habitats Directive) and Special Protection Areas (SPAs, Birds Directive) form this network. In France, the Directive was transposed in the Environmental Code. The operators (experts, site managers, etc.) shall assess the conservation status of the habitats of Community Interest within each Natura 2000 site. The management plan must include the results of the assessment. In order to achieve the objectives defined by the Directive, the French Ministry of the Environment has assigned the French National Museum of Natural History with the task of elaborating methods to assess the conservation status of all habitats of Community interest at the scale of Natura 2000 sites since 2008. The methods should be standardized, pragmatic, affordable and easy to apply. Several reports had been published about forest, agropastoral, coastal, marine and lagoonal habitats. The first method dealing with terrestrial wet habitats was published in 2013 . Then, other methods were set about standing waters, acid raised bogs,
\end{abstract}


KEY WORDS

Habitats Directive,

Natura 2000

Environmental Code, Site management. alkaline fens and Atlantic wet heathlands. Work is currently going on petrifying springs. The purpose is to create a series of grids to assess the conservation status of all wet habitats types. These grids are based on the assessment of three parameters, which are the surface, the structures and functions, as well as the deterioration factors. For each parameter, some criteria are selected and subsequently assessed with a set of indicators. For all the studies, a bibliographical research allows to preselect some indicators. Then, experts and site managers validate the lists of indicators to test in the field. The indicators are compared to an expert view on the conservation status of the habitats. The aim is to select only the indicators that correspond the most to the expert view. The testing phase leads to the definition of some thresholds to the indicators that correspond to the values at which the conservation status is considered to be "favourable" or "unfavourable". The testing phase takes place with the support of national botanical conservatories, conservatories of natural areas, other experts and site managers. Finally, statistical analyses allow to deliver initial results, which must be validated by the experts and the site managers before constituting a first proposal of assessment grid. The methods can evolve thanks to the feedback of users. Nowadays, $98 \%$ of all wet habitats of Community Interest present in mainland France are covered by at least a first proposal of indicators.

\section{INTRODUCTION}

La détérioration des écosystèmes est un problème majeur à l'échelle mondiale. Les principales menaces identifiées sont le changement d'utilisation des terres, l'exploitation directe des organismes, le changement climatique, les pollutions ou encore les espèces exotiques envahissantes (EEE) (Intergovernmental Science-Policy Platform on Biodiversity and Ecosystem Services 2019). Parmi eux, les écosystèmes d'eau douce, notamment les zones humides $(\mathrm{ZH})$, apparaissent comme les plus menacés. Du fait de leur importance écologique et de leur forte régression depuis des décennies, la convention de Ramsar de 1971 visait à engager les États membres à l'utilisation rationnelle des $\mathrm{ZH}$, à la bonne gestion des $\mathrm{ZH}$ d'importance internationale, et à la coopération internationale pour la protection des ZH transfrontières (Ramsar Convention Secretariat 2016). Plusieurs accords ont ensuite été conclus afin de préserver les écosystèmes dans le monde, dont le Programme des Nations unies pour l'environnement de 1972 (Programme des Nations Unies pour l'Environnement 1972) et la Convention sur la diversité biologique (Nations Unies 1992). Malgré tout, il ressort que les $\mathrm{ZH}$ sont particulièrement impactées à l'échelle mondiale. Seulement $13 \%$ de leur surface présente en 1700 perdurait en 2000 (Intergovernmental Science-Policy Platform on Biodiversity and Ecosystem Services 2019). Depuis 1900, $64 \%$ des ZH ont disparu (International Union for Conservation of Nature 2015). Les pertes plus récentes sont encore plus rapides: 0,8\% de la surface perdue par an entre 1970 et 2008 (Intergovernmental Science-Policy Platform on Biodiversity and Ecosystem Services 2019).

À l'échelle européenne, c'est la convention de Berne de 1979 qui fixe les bases de la conservation de la vie sauvage et des milieux naturels (Conseil de l'Europe 1979). La directive HabitatsFaune-Flore (DHFF) a par la suite été adoptée le 21 mai 1992 (Conseil des Communautés européennes 1992). Elle définit la notion d' «habitats naturels» comme étant «des zones terrestres ou aquatiques se distinguant par leurs caractéristiques géographiques, abiotiques et biotiques, qu'elles soient entièrement naturelles ou semi-naturelles» (Conseil des Communautés européennes 1992). Létat de conservation (EC) des habitats y est également défini comme «l'effet de l'ensemble des influences agissant sur un habitat naturel ainsi que sur les espèces typiques qu'il abrite, qui peuvent affecter à long terme sa répartition naturelle, sa structure et ses fonctions ainsi que la survie à long terme de ses espèces typiques [... ». La DHFF vise au maintien ou à la restauration dans un EC favorable des habitats et des espèces listés dans ses annexes. Ces habitats sont qualifiés "d'intérêt communautaire», car ils sont menacés de disparition et possèdent une aire de répartition naturelle restreinte ou réduite suite à leur régression. Ils abritent également des caractéristiques propres à l'une ou plusieurs des neuf régions biogéographiques (art. 1). Ces habitats d'intérêt communautaire (HIC) qualifiés de "génériques» sont décrits dans le Manuel d'interprétation des HIC EUR 28 (Fig. 1; Commission européenne 2013).

L'article 17, quant à lui, stipule que l'évaluation de l'EC des HIC listés en Annexe I et celle de l'ensemble des taxons des Annexes II, IV et V doit être effectuée tous les six ans au niveau biogéographique. Les États membres doivent également assurer la surveillance des HIC et des espèces d'intérêt communautaire (EIC) présents sur leur territoire (art. 11) (Conseil des Communautés européennes 1992). Suite à la DHFF, un outil européen de conservation des habitats, de la faune et de la flore a été créé, le réseau Natura 2000. Celui-ci vise à empêcher la perte de biodiversité, tout en considérant l'homme à part entière dans l'écosystème. Le réseau est composé de Zones de Protection spéciale (ZPS) au titre de la directive "Oiseaux» (Conseil des Communautés européennes 1979) et de Zones spéciales de conservation (ZSC) au titre de la DHFF. En 2017, le réseau couvrait $18,15 \%$ de la surface terrestre du territoire de l'Union européenne et $6 \%$ de la surface marine associée (Peters \& Von Unger 2017). La protection des aires est une politique de conservation classique s'appuyant sur le postulat qu'une aire protégée permet de conserver les habitats et les espèces menacés qui lui sont associés (Bartula et al. 2011).

La France métropolitaine recense $57 \%$ des HIC (131 types) listés en Annexe I de la DHFF et $17 \%$ des EIC (159 espèces) 


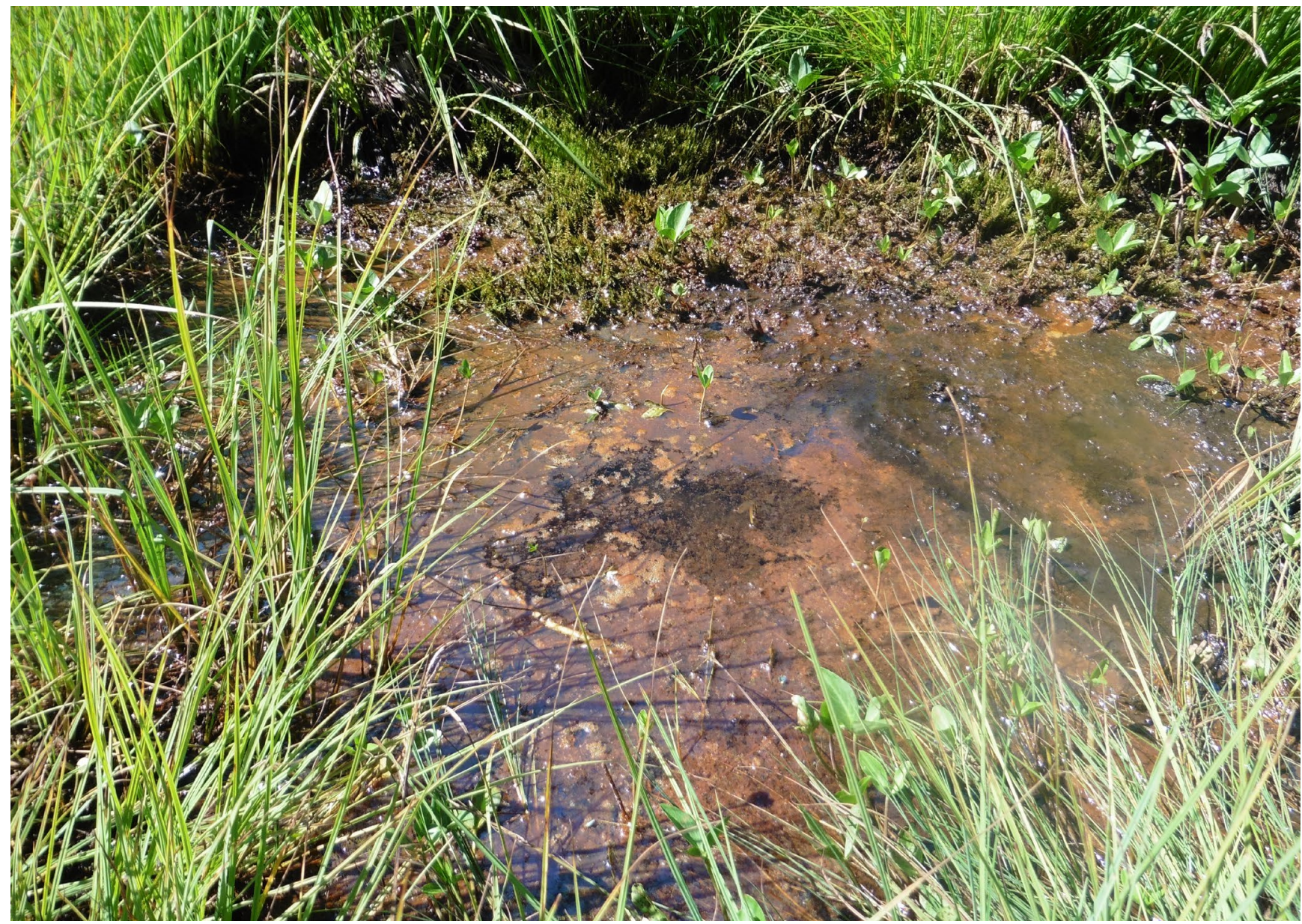

FIG. 1. - «Lacs et mares dystrophes naturels», habitat d'intérêt communautaire présent sur le territoire métropolitain (UE 3160). Crédit photo: M. Mistarz.

listées en Annexe II (inpn.mnhn.fr, dernière consultation le 12 janvier 2021). En 2017, le réseau Natura 2000 couvrait $12,9 \%$ du territoire métropolitain terrestre et un tiers de la surface marine, soit 1776 sites (ZPS et ZSC) (Peters \& Von Unger 2017). L'article R414-11 du Code de l'environnement (Anonyme 2008) notifie l'évaluation de l'EC des HIC et EIC pour chaque site Natura 2000. Il constitue ainsi une transposition de la DHFF dans le droit français. Les résultats de l'évaluation doivent être retranscrits dans le Document d'objectifs du site (DOCOB), qui permet de fixer les objectifs de conservation de la biodiversité et de gestion à l'échelle de chaque site.

Afin de répondre aux objectifs réglementaires, des réflexions sont engagées depuis 2008 dans le but de mettre en place des méthodes standardisées pour évaluer l'EC des HIC à l'échelle des sites Natura 2000, sur l'ensemble du territoire métropolitain. Plusieurs méthodologies ont été initiées ou mises en place, sur les habitats forestiers (Carnino 2009; Maciejewski 2016), les dunes non boisées du littoral atlantique (Goffé 2011), les habitats marins (Lepareur 2011; Le Floc'h 2015), les lagunes côtières (Lepareur et al. 2013, 2018) et les habitats agropastoraux (Maciejewski 2012; Maciejewski et al. 2013, 2015). La première méthode traitant des habitats humides a été publiée en 2013 (Viry 2013). S'en sont suivis depuis divers travaux.
Le fait que l'évaluation de l'EC des HIC soit effectuée par différents experts implique la production d'une méthode standardisée si l'on veut diminuer le risque d'interpréter la notion d'EC de différentes manières (Bottin et al. 2005). Le but recherché est donc la mise en place de méthodes pragmatiques, reproductibles et accessibles à tous les opérateurs. Par ailleurs, l'outil d'évaluation est une aide aux gestionnaires visant à définir les priorités d'intervention et les paramètres sur lesquels agir, de s'assurer de l'efficacité des mesures de gestion mises en œuvre et de définir le «bon EC». Les données recueillies à l'échelle des sites peuvent également participer à l'évaluation à l'échelle biogéographique prévue par l'article 17 de la DHFF (Conseil des Communautés européennes 1992).

La question à laquelle l'ensemble des méthodes doit répondre est donc la suivante: comment évaluer l'EC des HIC humides génériques sur l'ensemble du territoire métropolitain à l'échelle des sites Natura 2000?

Cet article vise à présenter le principe méthodologique de l'évaluation de l'EC des HIC humides à l'échelle des sites Natura 2000, les concepts étant exposés dans Maciejewski et al. (2016). L'élaboration de ces méthodes est ensuite détaillée. Enfin, un bilan des méthodes disponibles à ce jour pour évaluer l'EC des HIC humides est effectué. 


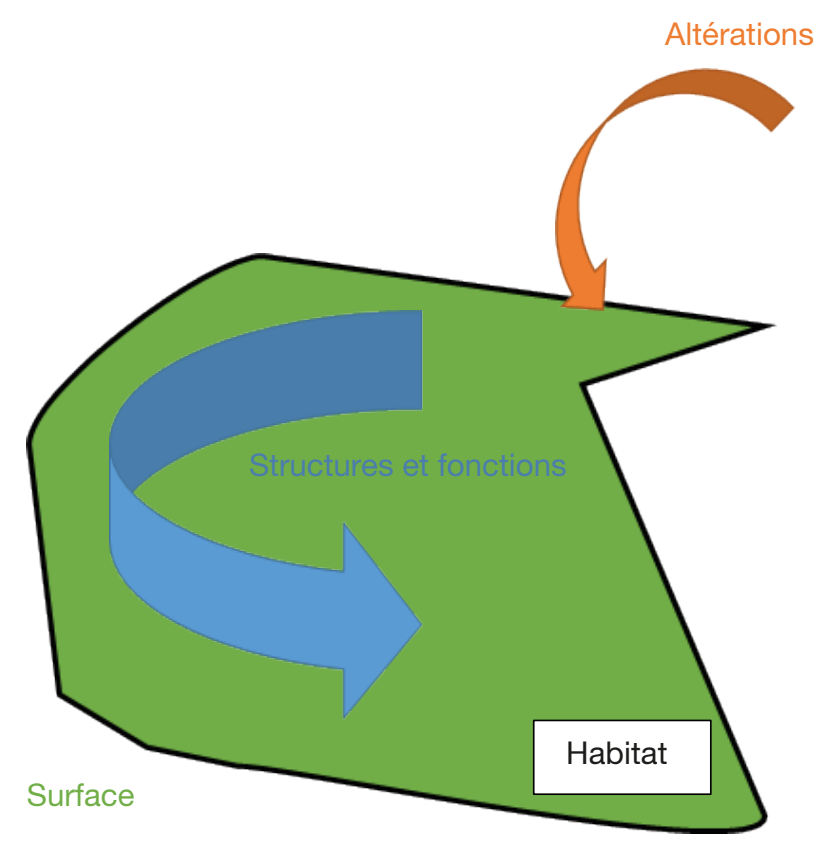

FIG. 2. - Schéma illustrant les trois paramètres pris en compte dans l'évaluation de l'état de conservation des habitats à l'échelle des sites Natura 2000.

\section{PRINCIPE MÉTHODOLOGIQUE DE L'ÉVALUATION À L'ÉCHELLE DES SITES}

\section{OBJETS ÉVALUÉS: DES HABITATS DYNAMIQUES \\ ET VARIABLES DANS LE TEMPS}

Les habitats évalués sont des HIC génériques (Commission européenne 2013). Sur le territoire métropolitain, les HIC humides ont été déclinés en habitats élémentaires dans les Cahiers d'habitats (Bensettiti et al. 2002) afin de prendre en compte leur variabilité sur le territoire. Cette variabilité peut être expliquée par plusieurs facteurs tels que l'aire biogéographique où l'habitat est présent, l'altitude, le type de substrat, la trophie de l'eau, etc. La majorité des méthodes proposées peut être appliquée à l'échelle de l'habitat générique, sauf exceptions.

Les habitats humides connaissent souvent des variabilités spatiales et temporelles importantes, aussi bien intra-annuelles qu'inter-annuelles. L'évaluation doit être effectuée à l'optimum de développement de la végétation afin de prendre en compte le maximum d'espèces constituant l'habitat. Cependant, il existe parfois une variabilité interannuelle forte des communautés, voire du niveau trophique dans lequel elles se développent. L'évaluation réalisée à un instant t doit, dans l'idéal, faire l'objet d'un suivi préalable, suivi fortement préconisé pour les végétations à éclipse (dont l'expression d'une année sur l'autre n'est pas systématique selon les conditions stationnelles). Il doit permettre de cibler les conditions climatiques favorables au développement de l'habitat (précipitations, températures, etc.).

\section{PARAMÈTRES, CRITÈRES, INDICATEURS}

Les grandes lignes de la démarche européenne pour l'évaluation de l'EC à l'échelle biogéographique (DG Environment 2017) sont reprises dans les méthodes développées à l'échelle des sites Natura 2000. Cependant, l'évaluation à l'échelle d'un site impose une adaptation des paramètres évalués à l'échelle biogéographique (aire de répartition naturelle de l'habitat, surface, structure et fonctions, perspectives futures). Ainsi, sont proposés trois paramètres pour l'évaluation de l'EC à l'échelle des sites que sont:

- la surface;

- les structures et fonctions;

- les altérations.

La surface est une composante spatiale en deux dimensions. Les structures et fonctions correspondent à l'ensemble des processus intrinsèques et nécessaires au maintien de l'habitat. Les altérations traduisent l'impact des facteurs externes, souvent d'origine anthropique (Fig. 2). Ces paramètres sont repris dans l'ensemble des méthodes mises en place. Un paramètre est défini ici comme un ensemble de critères permettant d'évaluer une même composante de l'EC (par exemple, les structures et fonctions). Ces critères constituent l'ensemble des processus et éléments clés qui vont influencer l'EC d'un habitat (composition floristique, dynamique hydromorphologique, atteintes lourdes, etc.).

Les critères sont évalués grâce à des indicateurs. Un indicateur peut être considéré comme un élément observable prenant soit une valeur qualitative, soit une valeur quantitative (par exemple, la présence ou le recouvrement de ligneux). Les indicateurs pris en compte dans l'évaluation de l'EC d'un habitat seront jugés "favorables» ou "défavorables, et hiérarchisés en fonction de l'importance de leur rôle dans la modification de l'EC de l'habitat (Maciejewski et al. 2016). L'ensemble des indicateurs permet ainsi d'indiquer le «bon» ou le «mauvais» EC de l'habitat en question.

UNE APPROCHE DE NOTATION GRADUELLE ET DÉGRESSIVE Dans un souci de cohérence et d'harmonisation des méthodologies, il a été décidé de conserver l'approche de notation graduelle et dégressive proposée pour l'évaluation de l'EC des habitats forestiers (Carnino 2009). Cette évaluation permet aux gestionnaires de prioriser les actions de restauration et/ ou gestion grâce à l'identification d'indicateurs importants pour la bonne caractérisation du fonctionnement de l'habitat.

La démarche globale de l'évaluation d'EC des habitats repose sur la comparaison de l'habitat étudié à une entité dont l'état est jugé optimal par l'opérateur pour ce type d'habitat (Maciejewski et al. 2016). Les valeurs des indicateurs observées sur le terrain sont comparées à des valeurs seuils. Chaque indicateur obtient une note, nulle ou négative. La somme des notes attribuées est additionnée à la note de 100. Ainsi, moins l'habitat est dégradé, plus la note sera élevée. La note finale est alors placée le long d'un gradient d'EC (Maciejewski et al. 2016). A noter que le seuil de 70 (passage des états altérés aux états favorables choisis) est un seuil ici arbitraire. Ce dernier peut être ajusté par l'opérateur à l'échelle du site Natura 2000 (Fig. 3).

Des notes positives peuvent être attribuées à certains indicateurs sous forme de bonus. On considère alors que l'indicateur, lorsqu'il est favorable, augmente la note de l'EC de l'habitat. En revanche, l'indicateur, s'il est défavorable, n'est 


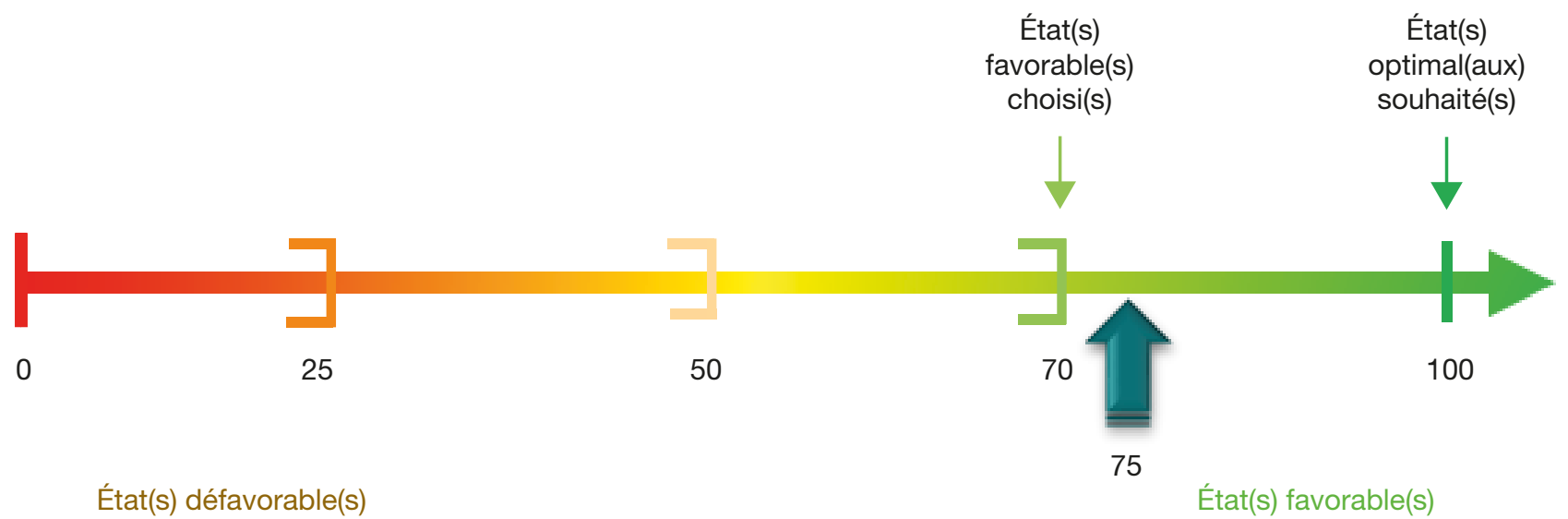

FIG. 3. - Positionnement de la note calculée par placette/polygone sur le gradient d'état de conservation (d'après Latour 2018).

pas pénalisant (aucun point n'est retiré). Cela peut, par exemple, correspondre à des indicateurs liés à la présence d'espèces faunistiques. L'idée est de considérer que l'absence des espèces faunistiques ciblées par la méthode n’indique pas systématiquement un mauvais EC de l'habitat. Puisque ce sont des espèces mobiles, elles peuvent être présentes sans être observées, à l'inverse des espèces floristiques. Des notes négatives peuvent également être attribuées sous forme de malus. Les malus peuvent correspondre à des processus n'étant pas strictement liés à l'EC des habitats comme, par exemple, l'apparition d'algues filamenteuses qui peut être liée soit à une pollution, soit à de fortes chaleurs. L'appréciation de la pertinence de ces indicateurs pour l'évaluation à l'échelle du site est laissée à l'opérateur.

\section{Plusieurs ÉCHELLES DE RELEVÉS POSSIBLES}

Les relevés d'indicateurs peuvent être effectués à plusieurs échelles : polygone, placette, écocomplexe, site Natura 2000, etc. Le polygone d'habitat est une entité relativement homogène sur le plan floristique, rattachée à l'habitat. Il est délimité par un changement dans la topographie, ou par l'existence de communautés végétales adjacentes, différentes de l'habitat, situées sur le même niveau topographique. L'ensemble du polygone est soumis à une même gestion (ou à l'absence de gestion). Le polygone cartographique peut être considéré comme un polygone sur lequel s'appliquera l'évaluation. La placette, elle, est une entité représentative du polygone d'habitat. Elle regroupe l'ensemble des caractéristiques majeures, observables à l'échelle du polygone (espèces floristiques, conditions stationnelles, etc.).

Une fois les indicateurs relevés aux différentes échelles, il existe plusieurs façons de passer de multiples évaluations stationnelles à une évaluation à l'échelle du site Natura 2000 : moyenne des notes de toutes les placettes, note globale en fonction de la proportion de placettes en état favorable ou dégradé, etc. Par ailleurs, des rendus complémentaires peuvent être réalisés : cartographie des placettes colorées selon leur EC, distribution des placettes sur le gradient d'EC, etc. Ces rendus complémentaires peuvent être envisagés par l'opérateur en fonction de ses objectifs.

\section{PROCESSUS GÉNÉRAL D’ÉLABORATION DES GRILLES D’ÉVALUATION}

\section{PRÉALABLE}

Les méthodes reposent sur un certain nombre d'indicateurs qualitatifs et quantitatifs. Un indicateur doit être simple à mesurer et relié au maintien des processus essentiels de l'habitat (Woodley \& Kay 1993). Dans le contexte de l'étude, il doit répondre rapidement à un facteur de dégradation. La récolte des données doit être peu coûteuse en temps et demander peu de compétences. Tous ces facteurs sont primordiaux si l'on veut mettre en place une méthode applicable sur le terrain et facilement reproductible. Les étapes d'élaboration d'une grille d'indicateurs pour évaluer l'EC des habitats humides sont résumées ci-dessous.

\section{RECHERCHE BIBLIOGRAPHIQUE ET PRÉSÉLECTION \\ D'INDICATEURS POTENTIELS}

Dans un premier temps, une recherche bibliographique approfondie permet de sélectionner une large liste d'indicateurs potentiels pour évaluer l'EC des habitats ciblés. Les objectifs de cette synthèse bibliographique sont, d'une part, d'établir une première liste non exhaustive d'indicateurs susceptibles d'évaluer les paramètres "Surface", "Structures et fonctions» et "Altérations». D'autre part, elle doit permettre de préétablir des seuils pour chaque indicateur présélectionné, c'est-à-dire les valeurs à partir desquelles l'indicateur passe de "favorable» à «non favorable». Les listes d'indicateurs potentiels peuvent reprendre les indicateurs utilisés dans des méthodes d'évaluation aux échelles locales en France (Klesczewski 2007; Pouvaret 2014; Lafon \& Le Fouler 2014), mais aussi en Europe (Mroz 2013; MuñozBarcia et al. 2019). De plus, de la littérature spécifique aux valeurs indicatrices des taxons caractéristiques des habitats ciblés est étudiée (Ellenberg 1988; Julve 1998), des documents phytosociologiques (Bardat et al. 2004; Bioret 2013; Delassus 2015) ou encore de la littérature spécifique aux processus qui conditionnent la présence des habitats et à leur gestion (Scheffer 2001; Ojeda 2009; Van Diggelen et al. 2015) (Tableau 1). 
TABLEAU 1. - Pré-liste d'indicateurs issus de la recherche bibliographique sur l'habitat des «Lacs eutrophes naturels avec végétation du Magnopotamion ou de l'Hydrocharition " (UE 3150) (d'après Mistarz \& Latour 2019).

\begin{tabular}{|c|c|c|c|c|}
\hline Paramètre & Critère & Indicateur & Informations apportées & Échelle \\
\hline Surface & Surface couverte & $\begin{array}{l}\text { Évolution de la surface couverte } \\
\text { Nombre de pièces d'eau où l'habitat } \\
\text { est présent }\end{array}$ & $\begin{array}{l}\text { Perspectives, pérennité de l'habitat, etc. } \\
\text { Perspectives, pérennité de l'habitat, etc. }\end{array}$ & $\begin{array}{l}\text { Site } \\
\text { Site }\end{array}$ \\
\hline \multirow[t]{9}{*}{$\begin{array}{l}\text { Structures } \\
\text { et fonctions }\end{array}$} & Caractéristiques de l'eau & Transparence de l'eau & $\begin{array}{l}\text { Hypereutrophisation, concentration en } \\
\text { matière organique en suspension, qualité } \\
\text { de l'eau, etc. }\end{array}$ & Placette \\
\hline & Sédimentation & Envasement (cm) & $\begin{array}{l}\text { Dynamique de comblement, susceptibilité } \\
\text { aux invasions, pérennité de l'habitat, etc. }\end{array}$ & Placette \\
\hline & \multirow[t]{5}{*}{ Composition floristique } & Recouvrement des hélophytes (\%) & $\begin{array}{l}\text { Dynamique de comblement, fermeture } \\
\text { du milieu, pérennité de l'habitat, } \\
\text { eutrophisation? }\end{array}$ & Placette \\
\hline & & Richesse spécifique & Intégrité, dynamique & Placette \\
\hline & & $\begin{array}{l}\text { Recouvrement des espèces } \\
\text { eutrophiles (\%) }\end{array}$ & Hypertrophisation/eutrophisation & Placette \\
\hline & & Recouvrement des EEE (\%) & $\begin{array}{l}\text { Capacité de résilience, qualité et } \\
\text { fonctionnement de l'habitat }\end{array}$ & Placette \\
\hline & & Indice F moyen & $\begin{array}{l}\text { Assèchement, drainage, dynamique et } \\
\text { pérennité de l'habitat }\end{array}$ & Placette \\
\hline & \multirow[t]{2}{*}{ Composition faunistique } & Macro-invertébrés (bonus) & Qualité de l'eau, fonctionnement général & Plan d'eau \\
\hline & & Amphibiens (bonus) & $\begin{array}{l}\text { Qualité de l'eau, fonctionnalité biologique, } \\
\text { connectivité des milieux }\end{array}$ & Plan d'eau \\
\hline \multirow[t]{2}{*}{ Altérations } & Atteintes lourdes & $\begin{array}{l}\text { Recouvrement des atteintes dont } \\
\text { l'impact peut être quantifié en } \\
\text { surface }\end{array}$ & $\begin{array}{l}\text { Fonctionnalité générale, connectivité, } \\
\text { capacité de résilience }\end{array}$ & Polygone \\
\hline & Atteintes diffuses & $\begin{array}{l}\text { Atteintes dont l'impact est } \\
\text { difficilement quantifiable en } \\
\text { surface }\end{array}$ & $\begin{array}{l}\text { Fonctionnement général, capacité de } \\
\text { résilience, pérennité de l'habitat }\end{array}$ & Site \\
\hline
\end{tabular}

VALIDATION ET SÉLECTION DES INDICATEURS À TESTER Les pré-grilles d'indicateurs issues de la recherche bibliographique sont ensuite présentées à des experts et gestionnaires lors d'un premier Comité de pilotage (COPIL). Les COPIL permettent d'échanger sur la pertinence et l'utilisation de certains indicateurs pour évaluer l'EC des habitats, leurs modalités d'application sur le terrain et les seuils potentiels. Ces échanges aboutissent à des modifications de la grille proposée initialement avec l'ajout, la suppression et/ ou le remaniement d'indicateurs. À noter que l'intégration d'indicateurs floristiques et faunistiques dans les méthodes d'évaluation de l'EC des milieux ouverts est un véritable enjeu (Carboni et al. 2015). Ce type d'indicateurs présente de nombreux avantages par rapport aux indicateurs physicochimiques (Menetrey et al. 2005). Ils intègrent les effets des variations des teneurs en nutriments sur de longues périodes, sont économiques et demandent un moindre effort de prospection que la mesure des paramètres physico-chimiques, qui ne fournit qu'une vision de la qualité chimique de l'eau à un instant $\mathrm{t}$.

\section{TEST DES INDICATEURS SUR LE TERRAIN}

Afin de tester les indicateurs retenus, des sites d'expérimentation sont choisis. L'objectif est de pouvoir prospecter l'ensemble de l'aire de répartition des habitats ciblés. L'intérêt est de pouvoir évaluer l'EC des habitats dans différents contextes (géographique, écologique, de gestion, etc.), et d'avoir suffisamment de recul pour proposer une méthode avec les indicateurs les plus pertinents aux échelles nationale et de l'habitat générique. Les sites sélectionnés sont des sites Natura 2000 ou non, disposant d'informations suffisantes sur les habitats ciblés (localisation notamment). Étant donné leur connaissance du terrain, les partenaires (conservatoires botaniques nationaux, chercheurs, conservatoires d'espaces naturels et autres structures gestionnaires) sont missionnés afin d'identifier les sites les plus adaptés. Idéalement, un site test doit présenter:

- un ou plusieurs DOCOB (cartographies, activités et leurs impacts sur l'ensemble du site, etc.);

- des polygones d'habitat en différents EC afin de calibrer la méthode sur l'ensemble des états rencontrés;

- des communautés végétales différentes pour un même HIC afin de pouvoir produire une méthode applicable à l'échelle de l'habitat générique;

- des polygones d'habitat relativement accessibles afin de maximiser le temps imparti à l'étude.

Les objectifs de la phase de terrain sont de:

- tester les indicateurs retenus lors du COPIL en les confrontant aux dires d'experts sur l'EC des placettes échantillonnées. Il s'agit ensuite de ne sélectionner que les indicateurs les mieux corrélés à l'avis d'expert;

- relever les atteintes et les données nécessaires à la mise en évidence des informations apportées par les indicateurs;

- mettre en évidence les corrélations et les redondances entre indicateurs.

Si les valeurs seuils des indicateurs sont prédéfinies grâce aux résultats de la recherche bibliographique ou à dire d'expert, la phase de terrain permet de les ajuster. Elle per- 

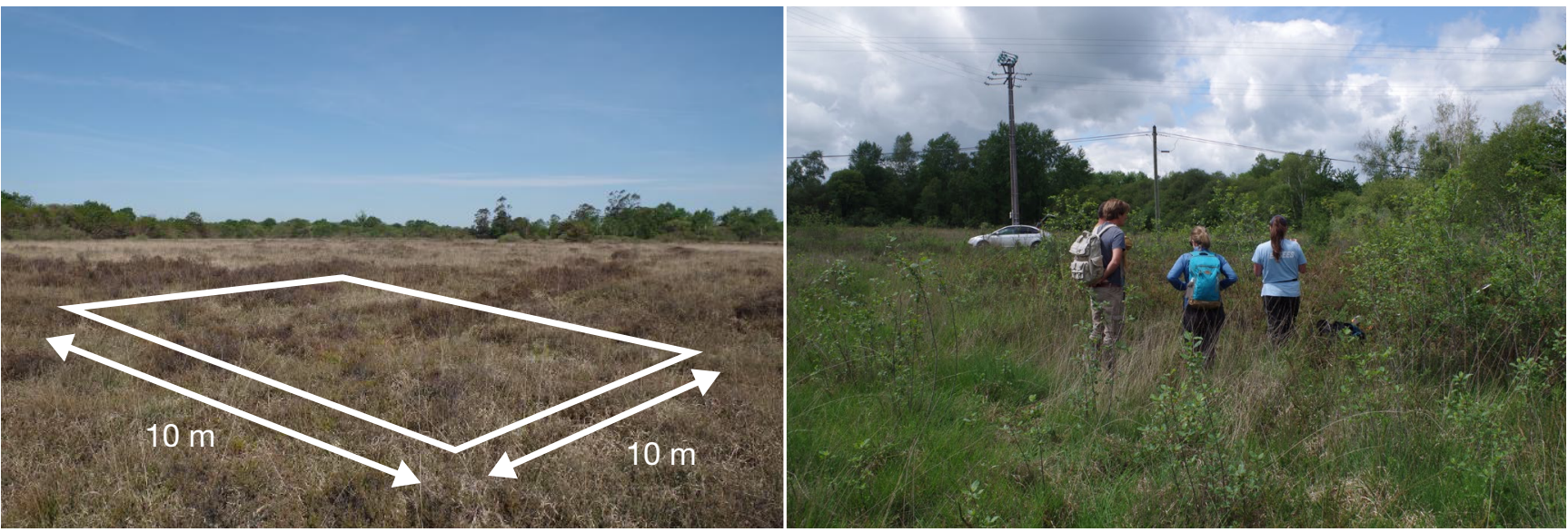

FIG. 4. - Exemples de relevés effectués sur des placettes délimitées de $100 \mathrm{~m}^{2}$ en landes humides (d'après Mistarz \& Grivel 2020). Crédits photos: M. Mistarz.

met de calibrer la méthode en attribuant les valeurs seuils et notes associées aux indicateurs retenus pour intégrer la méthodologie. Pour atteindre ces objectifs, sont réalisés sur les sites prospectés:

- une délimitation précise de l'habitat, ainsi qu'une géolocalisation des points de relevés;

- des photographies;

- des relevés phytosociologiques;

- des relevés d'indicateurs autres que ceux relatifs à la composition floristique;

- l'évaluation de l'EC de l'habitat à dire d'expert;

- un relevé des atteintes, le cas échéant (Fig. 4).

L'EC de chaque polygone d'habitat est renseigné comme étant «bon-optimal», «bon-correct», "altéré» ou "dégradé». Tous les commentaires et descripteurs de l'EC sont notés. Ces derniers permettent d'affiner l'EC de chaque polygone prospecté sous la forme «bon-correct + ", «bon-correct» ou "bon-correct -», afin d'avoir un large panel d'EC. Ainsi, la mise en évidence des relations entre indicateurs et avis d'expert n'en est que plus précise. L'avis d'expert permet également de fournir un ensemble de descripteurs permettant de définir les états de référence de l'habitat à l'échelle d'un site (états optimaux souhaités, favorables choisis, altérés et dégradés). Les concepts relatifs aux états de référence sont développés par Maciejewski et al. (2016).

\section{CONVERSION ET ANALYSE DES DONNÉES RÉCOLTÉES}

Une fois la phase de terrain achevée, les recouvrements des espèces contactées lors des relevés phytosociologiques sous forme de coefficients de Braun-Blanquet (1964) sont transformés en pourcentages de recouvrement. L'avis d'expert est transposé en variable qualitative ordinale, c'est-à-dire une variable qualifiant un état qui contient un ordre, ici lié à l'EC de l'habitat (bon-optimal, bon-correct, altéré, dégradé). Les stations échantillonnées sont ainsi finement hiérarchisées sur une échelle de 1 (très dégradé) à 12 (optimal).

Les analyses statistiques sont effectuées avec le logiciel $\mathrm{R}$ (The $\mathrm{R}$ Foundation 2020). Dans un premier temps, des analyses statistiques exploratoires sont réalisées afin de rendre compte de la variabilité et de l'amplitude écologique des habitats, ainsi que de faire un bilan de l'échantillonnage.

Des Analyses en Composantes principales, des Analyses des Correspondances multiples, des tests de corrélation ou encore des modèles linéaires, permettent de mettre en évidence les informations apportées par les indicateurs testés sur le terrain. Des tests de corrélations de Spearman sont effectués entre l'avis d'expert et chaque indicateur de type quantitatif (dont la valeur est numérique), ainsi qu'entre chaque indicateur de type quantitatif. Le coefficient de corrélation permet de juger de l'intensité de la relation entre deux variables. Il permet de savoir si une variable explique significativement la note d'avis d'expert, ou encore si les variables sont corrélées et apportent la même information. Pour les indicateurs de type qualitatif, des tests du Khi2 sont réalisés. Il est également possible de tester la corrélation entre l'avis d'expert et différentes combinaisons d'indicateurs via des modèles linéaires, l'objectif final étant de déterminer quelle combinaison d'indicateurs est la mieux corrélée à l'avis d'expert. Ces analyses permettent aussi de mettre en évidence des valeurs seuils pour les indicateurs qui n'en possédaient pas suite à la recherche bibliographique et à la phase de terrain.

\section{PRÉSENTATION ET VALIDATION DES RÉSULTATS} DE LA PHASE DE TEST SUR LE TERRAIN

Les résultats obtenus via analyses des données font l'objet d'une validation lors d'un second COPIL. Une fois les indicateurs et les valeurs seuils sélectionnés pour intégrer la grille finale, une note est obtenue par placette grâce à un calcul automatique. Cette note est confrontée à celle de l'avis d'expert par régression linéaire. Une boucle permet d'attribuer des notes associées aux différentes valeurs seuils pour chaque indicateur. Les notes qui correspondent au meilleur coefficient de détermination, c'est-à-dire à la meilleure prédiction entre la note calculée par placette et la note de l'avis d'expert, sont retenues. La démarche doit aboutir à une première version de grille de notation de l'EC (Tableau 2). 
TABLEAU 2. - Grille d'évaluation d'état de conservation des tourbières basses alcalines proposée en 2020 (UE 7230 ) (d'après Clément et al. 2020). Vert, favorable; jaune, entre favorable et moyen; orange, moyen; rouge, défavorable.

\begin{tabular}{|c|c|c|c|c|c|}
\hline Paramètre & Critère & Indicateur & Échelle & $\begin{array}{l}\text { Résultats } \\
\text { attendus }\end{array}$ & Notes \\
\hline Surface & Surface couverte & Évolution de la surface couverte par l'habitat & Site & $\begin{array}{c}\text { Progression, } \\
\text { stabilité } \\
\text { Régression }\end{array}$ & -10 \\
\hline \multirow[t]{5}{*}{$\begin{array}{l}\text { Structures et } \\
\text { fonctions }\end{array}$} & \multirow[t]{4}{*}{$\begin{array}{l}\text { Composition } \\
\text { floristique }\end{array}$} & Colonisation ligneuse : strate arbustive (1-7 m) (\%) & $\begin{array}{l}\text { Polygone ou } \\
\text { placette }\end{array}$ & $\begin{array}{c}<5 \\
5-25 \\
25-50 \\
>50\end{array}$ & $\begin{array}{r}0 \\
-15 \\
-30 \\
-45\end{array}$ \\
\hline & & Colonisation ligneuse : strate arborée (> $7 \mathrm{~m})(\%)$ & $\begin{array}{l}\text { Polygone ou } \\
\text { placette }\end{array}$ & $\begin{array}{c}<15 \\
15-75 \\
>75\end{array}$ & $\begin{array}{r}0 \\
-5 \\
-10\end{array}$ \\
\hline & & $\begin{array}{l}\text { Recouvrement des espèces indicatrices d'un } \\
\text { enrichissement trophique (\%) }\end{array}$ & Placette & $\begin{array}{c}<10 \\
10-30 \\
30-50 \\
>50\end{array}$ & $\begin{array}{r}0 \\
-20 \\
-40 \\
-60\end{array}$ \\
\hline & & Recouvrement des espèces liées au pâturage (\%) & Placette & $\begin{array}{c}<5 \\
5-25 \\
25-50 \\
>50\end{array}$ & $\begin{array}{r}0 \\
-15 \\
-30 \\
-45\end{array}$ \\
\hline & $\begin{array}{l}\text { Composition } \\
\text { faunistique }\end{array}$ & $\begin{array}{l}\text { Invertébrés (bonus): Vertigo geyeri (montagne), } \\
\text { V. angustior (plaine) }\end{array}$ & $\begin{array}{l}\text { Polygone ou } \\
\text { placette }\end{array}$ & Présence & 10 \\
\hline \multirow[t]{6}{*}{ Altérations } & \multirow[t]{4}{*}{ Atteintes lourdes } & \multirow[t]{4}{*}{ Recouvrement des atteintes quantifiables en surface } & \multirow[t]{4}{*}{ Polygone } & $\begin{array}{r}\text { Somme des } \\
\text { points }=0\end{array}$ & 0 \\
\hline & & & & $\begin{array}{l}\text { Somme des } \\
\text { points }=1\end{array}$ & -5 \\
\hline & & & & $\begin{array}{r}\text { Somme des } \\
\text { points }=2\end{array}$ & -10 \\
\hline & & & & $\begin{array}{r}\text { Somme des } \\
\text { points } \geq 3\end{array}$ & -15 \\
\hline & \multirow[t]{2}{*}{ Atteintes diffuses } & \multirow[t]{2}{*}{$\begin{array}{l}\text { Impact des atteintes difficilement quantifiables en } \\
\text { surface }\end{array}$} & \multirow[t]{2}{*}{$\begin{array}{l}\text { Site/Bassin } \\
\text { versant }\end{array}$} & $\begin{array}{l}\text { Nul ou faible } \\
\text { Moyen }\end{array}$ & $\begin{array}{r}0 \\
-10\end{array}$ \\
\hline & & & & Fort & -20 \\
\hline
\end{tabular}

\section{DISCUSSION SUR LES MÉTHODES PROPOSÉES}

\section{BILAN DES MÉTHODES DISPONIBLES POUR ÉVALUER}

\section{L'ÉTAT DE CONSERVATION DES HABITATS HUMIDES}

Le Tableau 3 présente l'ensemble des méthodes disponibles (versions les plus à jour) pour évaluer l'EC des habitats humides.

\section{DES MÉTHODES SIMPLES POUR UNE RÉALITÉ COMPLEXE}

La création de grilles d'indicateurs permettant l'évaluation standardisée des habitats humides sur l'ensemble du territoire métropolitain est essentielle afin de rendre compte de l'évolution des milieux. Ces grilles s'adressent à des opérateurs de site Natura 2000 disposant de moyens réalistes. En effet, l'application de ces méthodes doit minimiser les moyens nécessaires aux relevés et aux analyses. Il s'agit également de limiter les compétences requises en proposant des alternatives entre des indicateurs simples et des indicateurs demandant des connaissances expertes. Les listes d'espèces à prendre en compte dans l'évaluation se veulent également limitées. Ces méthodes constituent donc un compromis entre coûts (temps et compétences) et efficacité.

La validation des indicateurs passe par l'analyse de leur applicabilité sur le terrain, leur champ d'application et le degré de corrélation avec le type d'informations qu'ils sont supposés apporter (Paillet et al. 2015). Pour ce faire, l'évaluation du biais observateur est une étape essentielle de ce processus, surtout si les relevés de terrain sont voués à être effectués par des non spécialistes. Un indicateur peut être jugé pertinent lorsque le biais observateur est minimal, autrement dit s'il est facilement reproductible et que l'estimation de sa valeur est certaine. Les phases de terrain permettent de mettre en exergue les difficultés relatives à l'application de certains indicateurs et les biais relatifs à l'approche méthodologique proposée. L'évaluation des habitats à l'échelle des sites reste un exercice difficile et réducteur de la complexité des interactions entre les habitats et les composantes physiques du milieu dans lesquels ils se trouvent, ainsi qu'entre les habitats eux-mêmes (mosaïques par exemple) (Hardegen 2015). En effet, il n'est pas aisé d'allier les faibles compétences naturalistes souhaitées par les opérateurs à la précision scientifique requise pour évaluer l'EC des habitats au titre de la DHFF. Le budget et le temps souvent limités des opérateurs éliminent d'office certains indicateurs pourtant jugés pertinents, mais nécessitant du matériel coûteux pour leur mesure et beaucoup de temps pour leur détermination, leur analyse, etc. Enfin, peu d'indicateurs faunistiques intègrent encore ces grilles. Pourtant, ces derniers apparaissent essentiels pour traduire les interactions des habitats avec leur 
TABLEAU 3. - Bilan des méthodes les plus à jour élaborées par l'UMS Patrinat pour évaluer l'état de conservation des habitats humides d'intérêt communautaire en 2021. Gras*, habitats d'intérêt communautaire prioritaires.

\begin{tabular}{|c|c|c|c|}
\hline Type de milieux & Code UE & Dernière version & $\begin{array}{l}\text { Numéro de version pour les } \\
\text { habitats considérés }\end{array}$ \\
\hline Eaux dormantes & $\begin{array}{l}3110 \\
3120 \\
3130 \\
3140 \\
3150 \\
3160 \\
3170^{\star}\end{array}$ & $\begin{array}{c}\text { Mistarz \& Latour } 2019 \\
\text { Idem } \\
\text { Idem } \\
\text { Idem } \\
\text { Idem } \\
\text { Idem } \\
\text { Idem }\end{array}$ & $\begin{array}{l}1 \\
1 \\
1 \\
1 \\
1 \\
1 \\
1\end{array}$ \\
\hline Eaux courantes & $\begin{array}{l}3220 \\
3230 \\
3240 \\
3250 \\
3260 \\
3270 \\
3280 \\
3290\end{array}$ & $\begin{array}{rr}\text { Viry } 2013 \\
\\
\text { Idem } \\
\text { Idem } \\
\text { Idem } \\
\text { Mistarz } 2018 \\
\text { Viry 2013 } \\
\text { Mistarz 2018 } \\
\text { Idem }\end{array}$ & $\begin{array}{c}1 \\
1 \\
1 \\
1 \\
\text { Travail préliminaire } \\
\text { Travail préliminaire } \\
\text { Travail préliminaire } \\
\text { Travail préliminaire }\end{array}$ \\
\hline Landes & $\begin{array}{l}4010 \\
4020 *\end{array}$ & $\begin{array}{c}\text { Mistarz \& Grivel } 2020 \\
\text { Idem }\end{array}$ & $\begin{array}{l}1 \\
1\end{array}$ \\
\hline Mégaphorbiaies & 6430 & Maciejewski et al. 2015 & 1 \\
\hline Tourbières acides & $\begin{array}{l}7110^{\star} \\
7120 \\
7130 \\
7140 \\
7150\end{array}$ & $\begin{array}{l}\text { Epicoco \& Viry } 2015 \\
\text { Idem } \\
\text { Idem } \\
\text { Idem } \\
-\end{array}$ & $\begin{array}{c}\text { Travail préliminaire } \\
\text { Travail préliminaire } \\
\text { Travail préliminaire } \\
\text { Travail préliminaire } \\
\text { - }\end{array}$ \\
\hline Bas-marais calcaires & $\begin{array}{l}7210^{*} \\
7220^{*} \\
7230 \\
7240^{*}\end{array}$ & $\begin{array}{c}\text { Clément et al. } 2021 \\
\text { Idem } \\
\text { Idem } \\
\text { Idem }\end{array}$ & $\begin{array}{l}2 \\
1 \\
2 \\
2\end{array}$ \\
\hline Tourbières boisées & 91D0* & - & - \\
\hline
\end{tabular}

environnement, mais aussi pour qualifier l'état des processus intrinsèques aux habitats (rappelons que la notion d'habitat se rapporte à un biotope et une biocénose, laquelle est composée d'espèces végétales, mais aussi animales). Les travaux sur le lien espèces-habitats pourraient, à l'avenir, permettre de cibler des groupes faunistiques simples à prospecter et à déterminer.

\section{DES MÉTHOdES ÉVOLUTIVES ET ADAPTABLES}

Ces méthodes sont standardisées et applicables sur l'ensemble du territoire métropolitain, à l'échelle de l'habitat générique. Elles présentent les avantages d'être accessibles à tous, d'être reproductibles dans le temps et dans l'espace, de permettre la comparaison entre sites et d'identifier les efforts de gestion à fournir. Cependant, à chaque nouveau jeu de données récoltées sur le terrain, les indicateurs, valeurs seuils et notes sont recalibrés. La littérature récente permet aussi de faire évoluer les grilles proposées. Ces dernières doivent cependant être affinées par l'expérience de terrain et le retour des opérateurs.

Il est nécessaire d'adapter les méthodes proposées à l'échelle locale. Cela peut passer par l'ajout d'espèces au sein des listes prises en compte dans les différents indicateurs. En effet, une espèce considérée comme eutrophile à l'échelle locale peut ne pas être prise en compte dans un indicateur à l'échelle nationale. Ces grilles d'évaluation constituent une base de réflexion, que l'opérateur doit mener en amont de son application. Il s'agit de recontex- tualiser les indicateurs proposés à l'échelle de chaque site Natura 2000. D'autres méthodes existent pour évaluer l'EC des habitats humides en France (Prey \& Hauguel 2014; Wegnez 2017; Caillaud 2018). Ces dernières sont adaptées aux échelles locales. Clarifier les objectifs et les moyens permet de choisir l'outil adéquat.

\section{LE CHOIX DES ÉTATS DE RÉFÉRENCE}

AUX ÉCHELLES NATIONALE ET DE L'HABITAT GÉNÉRIQUE Le principe de l'évaluation implique de choisir les états de référence, à partir desquels on considère que l'habitat est en EC favorable, altéré ou dégradé. Si la littérature scientifique peut aider à construire ces différents états en se basant sur un certain nombre de critères et indicateurs, le nombre de travaux et publications sur le sujet n'en reste pas moins limité. La phase de test des indicateurs sur le terrain peut alors aider à construire ces différents états. Ceci implique de visiter une grande diversité de sites où l'habitat générique est bien identifié, afin d'avoir un jeu de données représentatif de sa variabilité géographique, et des communautés qui le composent au sein du territoire. C'est pourquoi plusieurs descripteurs sont proposés pour décrire les états de référence des habitats humides. Il s'agit de garder à l'esprit que ces états doivent être établis par l'opérateur à l'échelle de son site Natura 2000. Une fois ces états construits, le seuil à partir duquel l'habitat passe d'un état altéré à favorable peut être fixé. Au sein d'un même site, plusieurs états favorables peuvent être envisagés pour un seul habitat générique. 


\section{CONCLUSION}

Le travail mené par l'UMS Patrinat et les travaux conduits aux échelles locales constituent les approches de mise en place et d'application de méthodes standardisées d'évaluation de l'EC des HIC humides sur l'ensemble de la France métropolitaine. L’analyse de la littérature, les COPIL, ainsi que les phases de terrain successives permettent de mettre en avant des indicateurs potentiellement pertinents dans une démarche d'évaluation nationale. Ces études aboutissent à la production de guides méthodologiques à destination des opérateurs.

Les versions des différentes grilles proposées aujourd'hui sont plus ou moins abouties selon les types d'habitats. Les méthodes jugées tout à fait valides et pertinentes par les différentes parties prenantes ayant participé à leur réalisation se veulent évolutives. Les grilles seront remaniées après chaque nouvelle phase de test et/ou retours d'expérience des utilisateurs, si jugé pertinent. Un équilibre entre précision scientifique et faisabilité est primordial pour que ces méthodes soient à la fois valides et applicables par les opérateurs Natura 2000. Ce travail n'en reste pas moins essentiel si l'on souhaite rendre compte de l'évolution des habitats français au sein du réseau européen.

\section{Remerciements}

Je souhaiterais remercier tous les stagiaires qui se sont succédés depuis 2017 et qui ont activement contribué à l'élaboration des méthodes sur les bas-marais, les eaux dormantes et les landes humides: Hugo Clément, Aleksander Miedziejewski, Manon Latour, Julie Garcin, Mathilde Reich, Lisa Grivel et François Botcazou. Merci également à Lise Maciejewski (UMS Patrinat/AgroParisTech) et à Adèle Rauzier (PN du Mercantour) pour leur relecture attentive. Enfin, merci à tous les partenaires, experts et gestionnaires, qui ont travaillé avec nous sur la thématique depuis 2016: Olivier Argagnon, Henri Michaud, Benoît Offerhaus (CBNméd), Laurent Sorba, Christophe Panaiotis, Laetitia Hugot, Marie-Laurore Pozzo di borgo, Paula Spinosi, Viviane Sorba (OEC), Jérémy Lebrun, Nicolas Caron, Thomas Cheyrezy, Marie-Hélène Guislain (CEN Picardie), Adrien Messean (CEN-Hautsde-France), Christophe Galet (SMOA), Jean-Christophe Hauguel, Raphaël Coulombel (CBNBl), Laurent Chabrol, Mickaël Mady, Nicolas Guillerme (CBNMC), Guillaume Choisnet (COENOSE), Leslie Ferreira, Julien Mondion, Sophie Auvert, Ophélie Beslin, Sébastien Filoche, Guillaume Billod, Frédéric Hendoux, Emilie Weber, Jérôme Wegnez, Olivier Bardet, Florient Desmoulin, Marie Leblanc (CBNBP), Jérémie Van Es, Thomas Legland, Thomas Sanz, Gilles Pache, David Paulin, Sylvain Abdulhak, Luc Garraud (CBNA), Florent Arthaud (USMB), Ludovic Olicard, Catherine BrauNogué (CBNPMP), Rémi Collaud (CBNFC-ORI), Marie Liron, Pauline D’Adamo (PNRPC), David Bécu, Michel Billod, Guillaume Geneste, Romaric Leconte (CEN CA), Dominique Lopez-Pinot, Olivier Billant (Asters), Anthony Garcia (CEN RA), Virginie Bourgoin, Manuel Bouron,
Jérôme Porteret, Philippe Freydier (CEN Savoie), Marie-José Trivaudey-Vergon (DREAL Bourgogne-Franche-Comté), Pierre Durlet (PNR du Haut-Jura), Vincent Gaudillat, Rémy Poncet, Jean-Christophe de Massary, Pascal Dupont, Isabelle Witté (UMS Patrinat), Jean-Jacques Boutteaux, Matthieu Perrez, Frédéric Brendel (ONF), Cécile Guérin (Marais de Lavours), Fabrice Darinot (Marais de Lavours), Charlotte Horon (SIGEA), Franck Quénault, Charlotte Dubreuil (SIAEBVELG), Vincent Labourel (CEN Aquitaine), Anthony Le Fouler, Pierre Lafon, Marie Caillaud, Rémi Guisier (CBNSA), Sébastien Lecuyer (La Roselière), François Sargos (SEPANSO), Valentine Dupont, Yann Sellier (GEREPI), Arnaud Duranel (University College London), Pierre Goubet (Cabinet Pierre Goubet), Guillaume Doucet (CEN B), Renaud Jaunatre (INRAE), Nadège Popoff (INRAE), Loïc Delassus, Marie Goret (CBNB), Gwenhael Perrin (UBO), Emmanuel Holder (Bretagne Vivante), Aline Bifolchi (Association de Mise en Valeur des sites naturels de Glomel), Arnaud Six (PNR Périgord-Limousin), Christophe Bach (CNPF), Julia Combrun (PNR Normandie-Maine), Maiwenn Le Rest (CPIE du Cotentin), Jérôme FouertPouret (PNR des Landes de Gascogne), Bernard Clément, Laura Granato (CEN PACA), Lydie Labrosse (SM3A), Florence Niel (PNR du Vercors), Céline Przysiecki (PNR $\mathrm{du}$ Vexin français), Nolwenn Quilliec (RNN Coteaux de la Seine), Tony Rulence (CG60), Loïc Raspail (Communauté de communes des Balcons du Dauphiné), Violaine Meslier (AGRENABA), Raphaël Quesada (Lo Parvi), Nina Schoen (Communauté de communes La Domitienne), Claire Betbeder (Communauté de communes des Grands Lacs), Chloé Rouzeyre (Communauté d'agglomération Hérault Méditerranée), Julien Azéma (Communauté d'agglomération Hérault Méditerranée), Sophie Drai (Communauté d'agglomération Hérault Méditerranée) et Nicolas Thomas (Communauté d'agglomération Var Estérel Méditerranée).

\section{RÉFÉRENCES}

AnOnYme 2008. - Article R414-11 du Code de l'environnement modifié par décret n ${ }^{\circ} 2015-959$ du 31 Juillet 2015-Art. 1. http:// www.legifrance.gouv.fr, dernière consultation le 27 avril 2020.

Bardat J., Bioret F., Botineau M., Boullet V., Delpech R., Géhu J.-M., Haury J., Lacoste A., Rameau J.-C., Royer J.-M., Roux G. \& Touffet J. 2004. - Prodrome des végétations de France. Muséum national d'Histoire naturelle (Coll. Patrimoines naturels; 61), Paris, $171 \mathrm{p}$.

Bartula M., Stojšić V., Perić R. \& Kitnæes K. S. 2011. — Protection of Natura 2000 habitat types in the Ramsar site "Zasavica special nature reserve» in Serbia. Natural Areas Journal 31 (4): 349-357. https://doi.org/10.3375/043.031.0405

Bensettiti F., Gaudillat V. \& Haury J. 2002. - "Cahiers d'habitats" Natura 2000. Connaissance et gestion des habitats et des espèces d'intérêt communautaire. Tome 3 : Habitats humides. La Documentation française, Paris, 457 p.

BIORET F. 2013. - Le prodrome des végétations de France, premier référentiel phytosociologique national. E.R.I.C.A. 26, 6 p.

Bottin G., Etienne M., Verté P. \& Mahy G. 2005. - Methodology for the elaboration of Natura 2000 sites designation acts in the Walloon Region (Belgium): calcareous grasslands in the Lesse-and-Lomme area. Biotechnologie, Agronomie, Société 
et Environnement 9 (2): 101-110.

Braun-BlanQuet J. 1964. - Pflanzensoziologie. Grundzüge der Vegetationskunde. Springer, Wien, New-York, 865 p.

CaILlaud M. 2018. - État de conservation des habitats d'intérêt communautaire rivulaires des étangs arrière-littoraux d'Aquitaine. Méthode d'évaluation par indices. Rapport de stage. Conservatoire botanique national Sud-Atlantique, DREAL Nouvelle-Aquitaine, Université de Bretagne occidentale, Brest, 38 p.

Carboni M., Dengler J., Mantilla-Contreras J., Venn S. \& TÖRÖK P. 2015. - Conservation value, management and restoration of Europe's semi-natural open landscapes. Hacquetia 14 (1): 5-17. https://doi.org/10.1515/hacq-2015-0017

CARNINO N. 2009. - État de conservation des habitats d'intérêt communautaire à l'échelle du site. Méthode d'évaluation des habitats forestiers. Muséum national d'Histoire naturelle, Office national des Forêts, Paris, 49 p.

Clément H., Reich M., Mistarz M. \& Garcin J. 2020. Evaluation de l'état de conservation des bas-marais calcaires d'intérêt communautaire. Cahiers d'évaluation à l'échelle des sites Natura 2000. Version 1. UMS Patrinat - OFB/CNRS/ MNHN, Paris, 183 p.

Clément H., Reich M., Botcazou F., Mistarz M. \& Garcin J. 2021. - Évaluation de l'état de conservation des bas-marais calcaires d'intérêt communautaire. Cahiers d'évaluation à l'échelle des sites Natura 2000. Version 2. UMS Patrinat - OFB/CNRS/ MNHN, Paris, 185 p.

COMMISSION EUROPÉENNE 2013. - Natura 2000. Interpretation manual of European habitats. EUR 28. Commission européenne, Bruxelles, $144 \mathrm{p}$.

CONSEIL DE L'EUROPE 1979. - Convention relative à la conservation de la vie sauvage et du milieu naturel de l'Europe. Série des traités européens - n¹04. Conseil de l'Europe, Berne, 10 p.

Conseil des Communautés européennes 1979. — Directive 79/409/CEE du Conseil concernant la conservation des oiseaux sauvages. Journal officiel des Communautés européennes L103.

Conseil des Communautés EuropéEnnes 1992. - Directive 92/43/CEE du Conseil du 21 Mai 1992 concernant la conservation des habitats naturels ainsi que de la faune et de la flore sauvages. Journal officiel de l'Union européenne, L206.

Delassus L. 2015. - Guide de terrain pour la réalisation des relevés phytosociologiques. Conservatoire botanique nationa de Brest, 25 p.

DG EnVIRONMENT 2017. - Reporting under Article 17 of the Habitats Directive: Explanatory Notes and Guidelines for the Period 2013-2018. DG Environment, Bruxelles, 187 p.

EllenberG H. 1988. - Vegetation Ecology of Central Europe. Cambridge University Press, Cambridge, 758 p.

EPICOCO C. \& VIRY D. 2015. - Etat de conservation des habitats tourbeux d'intérêt communautaire. Méthode d'évaluation à l'échelle du site Natura 2000. Rapport préliminaire. Version 1. Rapport SPN 2015-57. Service du Patrimoine naturel/Muséum national d'Histoire naturelle, Office national de l'Eau et des Milieux aquatiques, Paris, 111 p.

GOFFÉ L. 2011. - État de conservation des habitats d'intérêt communautaire des dunes non boisées du littoral atlantique. Méthode d'évaluation à l'échelle du site Natura 2000. Version 1. Rapport SPN 2011-18. Muséum national d'Histoire naturelle, Office nationa des Forêts, Conservatoire botanique national de Brest, 67 p.

HARDEGEN M. 2015. - Natura 2000 en Bretagne: habitats d'intérêt communautaire terrestres et d'eau douce. Bilan des connaissances: interprétation, répartition, enjeux de conservation. Direction régionale de l'Environnement, de l'Aménagement et du Logement Bretagne, Rennes; Conservatoire botanique national, Brest, 242 p.

INTERGOVERNMENTAL SCIENCE-POLICY PlATFORM ON BIODIVERSITY AND ECOSYSTEM SERVICES 2019. - Summary for Policymakers of the Global Assessment Report on Biodiversity and Ecosystem Services of the Intergovernmental Science-Policy Platform on Bio- diversity and Ecosystem Services. IPBES secretariat, Bonn, $56 \mathrm{p}$ INTERNATIONAL UNION FOR CONSERVATION OF NATURE 2015. IUCN-Ramsar Collaboration - Supporting the Wise Use of Wetlands. IUCN, Gland, 9 p.

Julve P. 1998. - Baseflor. Index botanique, écologique et chorologique de la flore de France. Version: 6 juillet 2016. http://philippe. julve.pagesperso-orange.fr/catminat.htm, dernière consultation le 5 janvier 2021.

KLESCZEWSKI M. 2007. - Élaboration de critères d'évaluation de l'état de conservation des habitats naturels du Parc national des Cévennes. Conservatoire d'espaces naturels du LanguedocRoussillon, Montpellier, 65 p.

LAFON P. \& LE FOUler A. 2014. - Évaluation et suivi de l'état de conservation des landes et tourbières d'Aquitaine-Méthode et mise en place. Conservatoire botanique national Sud-Atlantique, Direction régionale de l'Environnement, de l'Aménagement et du Logement Aquitaine, Audenge, 19 p.

Latour M. 2018. - Méthode d'évaluation de l'état de conservation des habitats d'intérêt communautaire à l'échelle des sites Natura 2000: "Eaux oligotrophes très peu minéralisées sur sols généralement sableux de l'ouest méditerranéen à Isoetes spp. " (UE 3120) et "Mares temporaires méditerranéennes" (UE 3170*). UMS Patrinat, Paris ; Conservatoire botanique national méditerranéen de Porquerolles, Hyères ; Università di Corsica - Pasquale Paoli, Paris, 62 p.

LE FloC'H M. 2015. — Phase préliminaire de l'élaboration de la méthode d'évaluation de l'état de conservation de l'habitat d'intérêt communautaire "Estuaires" (UE 1130) de la façade Manchel Atlantique. Service du Patrimoine naturel/Muséum national d'Histoire naturelle, Paris, 69 p.

LEPAREUR F. 2011. - Évaluation de l'état de conservation des habitats naturels marins à l'échelle d'un site Natura 2000. Guide méthodologique. Version 1. Rapport SPN 2011-3. Muséum national d'Histoire naturelle, Paris, $55 \mathrm{p}$.

Lepareur F., Bertrand S., PAPUGA G. \& Richeux M. 2013. État de conservation de l'habitat 1150 "Lagunes côtières". Méthode d'évaluation à l'échelle du site Natura 2000. Guide d'application. Version 1. Rapport SPN 2013-14. Service du Patrimoine naturel/ Muséum national d'Histoire naturelle, Paris, 107 p.

Lepareur F., Bertrand S., Morin E., Le Floc'h M., Barre N., GARRIDO M., RIERA L. \& MAUClERT V. 2018. — État de conservation des "Lagunes côtières" d'intérêt communautaire (UE 1150*). Méthode d'évaluation à l'échelle du site - Guide d'application (Version 2). Rapport UMS Patrinat. Muséum national d'Histoire naturelle, Pôle-relais lagunes méditerranéennes, Paris, 73 p.

MACIEJEWSKI L. 2012. - Etat de conservation des habitats agropastoraux d'intérêt communautaire. Méthode d'évaluation à l'échelle du site Natura 2000. Rapport d'étude. Version 1. Rapport SPN 2012-21. Service du Patrimoine naturel/Muséum national d'Histoire naturelle, Paris, 119 p.

MACIEJEWSKI L. 2016. - État de conservation des habitats forestiers d'intérêt communautaire. Évaluation à l'échelle du site Natura 2000. Version 2. Tome 1. Définitions, concepts et éléments d'écologie. Rapport SPN 2016-75. Service du Patrimoine naturel/Muséum national d'Histoire naturelle, Paris, $62 \mathrm{p}$.

Maciejewski L., Seytre L., Van Es J., Dupont P. \& BenMiMOUN K. 2013. - État de conservation des habitats agropastoraux d'intérêt communautaire. Méthode d'évaluation à l'échelle du site Natura 2000. Guide d'application. Version 2. Rapport SPN 2013-16. Service du Patrimoine naturel/Muséum national d'Histoire naturelle, Paris, 179 p.

Maciejewsiki L., Seytre L., VAn Es J. \& Dupont P. 2015. État de conservation des habitats agropastoraux d'intérêt communautaire. Méthode d'évaluation à l'échelle du site Natura 2000. Guide d'application. Version 2. Rapport SPN 2015-43. Service du Patrimoine naturel/Muséum national d'Histoire naturelle, Paris, 194 p.

Maciejewski L., Lepareur F., Viry D., Bensettiti F., Puis- 
SAuve R. \& Touroult J. 2016. - État de conservation des habitats: propositions de définitions et de concepts pour l'évaluation à l'échelle d'un site Natura 2000. Revue d'Écologie (Terre et Vie) 71 (1): 3-20.

Menetrey N., Sager L., Oertli B. \& Lachavanne J-B. 2005 . Looking for metrics to assess the trophic state of ponds. Macroinvertebrates and amphibians. Aquatic Conservation: Marine and Freshwater Ecosystems 15 (6): 653-664. https://doi.org/10.1002/ aqc. 746

MistarZ M. 2018. - Guide méthodologique pour la surveillance des habitats d'eau courante d'intérêt communautaire en vue du rapportage 2018 (Art. 17 DHFF). UE 3260: Rivières des étages planitiaire à montagnard avec végétation du Ranunculion fluitantis et $d u$ Callitricho-Batrachion. UE 3280: Rivières permanentes méditerranéennes du Paspalo-Agrostidion avec rideaux boisés riverains à Salix et Populus alba. UE 3290: Rivières intermittentes méditerranéennes du Paspalo-Agrostidion. Rapport Patrinat 2018-2. UMS Patrinat - AFB/MNHN/CNRS, Paris, 61 p.

Mistarz M. \& Grivel L. 2020. - Évaluation de l'état de conservation des landes humides d'intérêt communautaire. Cahiers d'évaluation à l'échelle des sites Natura 2000. Version 1. UMS Patrinat - OFB/CNRS/MNHN, Paris, 88 p.

Mistarz M. \& Latour M. 2019. - Etat de conservation des habitats des eaux dormantes d'intérêt communautaire. Méthodes d'évaluation à l'échelle des sites Natura 2000. Cahiers d'évaluation. UMS Patrinat - AFB/CNRS/MNHN, Paris, 252 p.

Mroz W. 2013. - Monitoring of Natural Habitats. Methodological Guide for Five Natural Habitats: 3110, 6210, 6520, 7230 and 9180. GIOŚ, Warszawa, $97 \mathrm{p}$

Muñoz-Barcia C. V., Lagos L., Blancos-Arias C. A., DíazVARELA R. \& FAGÚNDEZ J. 2019. - Habitat quality assessment of Atlantic wet heathlands in Serra do Xistral, NW Spain. Cuadernos de Investigación Geográfica 45 (2): 533-549. http:// doi.org/10.18172/cig.3628

NATIONS UNIES 1992. - Convention sur la diversité biologique. Nations Unies, Rio de Janeiro, 30 p.

OJEDA F. 2009. - 4020 Brezales húmedos atlánticos de Erica ciliaris $(*)$. Bases ecológicas preliminares para la conservación de los tipos de hábitat de interés comunitario en España. Ministerio de Medio Ambiente, y Medio Rural y Marino, Madrid, 49 p.

Paillet Y., Coutadeur P., Vuidot A., Archaux F. \& Gosselin F. 2015. - Strong observer effect on tree microhabitats inventories: A case study in a French lowland forest. Ecological Indicators 49: 14-23. https://doi.org/10.1016/j.ecolind.2014.08.023

Peters J. \& Von Unger M. 2017. - Peatlands in the EU Regulatory Environment. Federal Agency for Nature Conservation,
Bonn, $103 \mathrm{p}$.

POUVARET S. 2014. - Mise en ceuvre du document d'objectif du site Natura 2000 "Val d'Allier Alagnon" (FR 830 1038) - Animation de la mise en æuvre, compte rendu d'exécution - Évaluation de l'état de conservation de l'habitat d'intérêt communautaire 3150 "Lacs eutrophes naturels avec végétation du Magnopotamion ou de l'Hydrocharition ". Natura 2000, Direction régionale de l'Environnement, de l'Aménagement et du Logement Auvergne, Clermont-Ferrand; Conservatoire d'espaces naturels d'Auvergne-Riom, Riom, 27 p.

Prey T. \& Hauguel J.-C. 2014. - Évaluation de l'état de conservation des habitats d'intérêt communautaire en région Picardie. Analyse des méthodologies et des résultats obtenus. Centre régional de phytosociologie/Conservatoire botanique national de Bailleul; Direction régionale de l'Environnement, de l'Aménagement et du Logement, Amiens, $96 \mathrm{p}$.

Programme des Nations Unies pour l'Environnement 1972. - Declaration of the United Nations Conference on the Human Environment. United Nations, Stockholm, 81 p.

Ramsar Convention Secretariat 2016. - An Introduction to the Ramsar Convention on Wetlands. Fifth ed. Ramsar Handbooks, Ramsar, $107 \mathrm{p}$.

SCHEFFER M. 2001. - Alternative Attractors of Shallow Lakes. The Scientific World Journal 1: 254-63. https://doi.org/10.1100/ tsw. 2001.62

The R Foundation 2020. - The R Project for Statistical Computing. https://www.r-project.org, dernière consultation le 21 septembre 2020 .

Van Diggelen J. M. H., Bense I. H. M., Brouwer E., Limpens J., Van Schie J. M. M., Smolders A. J. P. \& Lamers L. P. M. 2015. - Restoration of acidified and eutrophied rich fens: Long-term effects of traditional management and experimental liming. Ecological Engineering 75: 208-216. https://doi. org/10.1016/j.ecoleng.2014.12.006

VIRY D. 2013. - État de conservation des habitats humides et aquatiques d'intérêt communautaire. Méthode d'évaluation à l'échelle du site Natura 2000. Rapport d'étude. Version 1. Rapport SPN 2013-12. Service du Patrimoine naturel/Muséum national d'Histoire naturelle, Office national de l'Eau et des Milieux aquatiques, Paris, 83 p.

Wegnez J. 2017. — Les landes d'Île-de-France. Deuxième partie. Protocole d'évaluation de l'état de conservation des habitats 4010 et 4030. Version 2. Conservatoire botanique national du Bassin parisien/Muséum national d'Histoire naturelle, Délégation Île-de-France, Paris, 27 p.

WoOdLEY S. \& KAY J. 1993. - Ecological Integrity and the Management of Ecosystems. Taylor \& Francis, Ottawa, 221 p. 